\title{
Effect of Sodium Lauryl Sulfate (SLS) Applied as a Patch on Human Skin Physiology and Its Microbiota
}

\author{
Sabrina Leoty-Okombi ${ }^{1, *}$, Florence Gillaizeau ${ }^{2}$, Sébastien Leuillet ${ }^{2}$, Benoit Douillard ${ }^{2}$, \\ Sophie Le Fresne-Languille ${ }^{2}$, Thomas Carton ${ }^{2}$, Alessandra De Martino ${ }^{2}$, Philippe Moussou ${ }^{1}$, \\ Catherine Bonnaud-Rosaye ${ }^{1}$ and Valérie André ${ }^{1}$
}

1 BASF Beauty Care Solutions France, 32 rue Saint Jean de Dieu, 69007 Lyon, France; philippe.moussou@basf.com (P.M.); catherine.bonnaud-rosaye@basf.com (C.B.-R.); valerie.andre-frei@basf.com (V.A.)

2 Biofortis, Mérieux NutriSciences Company, 3 Route de la Chatterie, Saint-Herblain, 44800 Nantes, France; florence.gillaizeau@mxns.com (F.G.); sebastien.leuillet@mxns.com (S.L.); benoit.douillard@mxns.com (B.D.); sophie.le.fresne@mxns.com (S.L.F.-L.); Thomas.carton@mxns.com (T.C.); alessdemartino@gmail.com (A.D.M.)

* Correspondence: sabrina.leoty-okombi@basf.com; Tel.: +33-472-766-000

\section{check for}

updates

Citation: Leoty-Okombi, S.; Gillaizeau, F.; Leuillet, S.; Douillard, B.; Le Fresne-Languille, S.; Carton, T.; De Martino, A.; Moussou, P.; Bonnaud-Rosaye, C.; André, V. Effect of Sodium Lauryl Sulfate (SLS) Applied as a Patch on Human Skin Physiology and Its Microbiota. Cosmetics 2021, 8, 6. https://doi.org/ 10.3390/cosmetics8010006

Received: 4 December 2020 Accepted: 24 December 2020 Published: 6 January 2021

Publisher's Note: MDPI stays neutral with regard to jurisdictional clai$\mathrm{ms}$ in published maps and institutional affiliations.

Copyright: (C) 2021 by the authors. Licensee MDPI, Basel, Switzerland. This article is an open access article distributed under the terms and conditions of the Creative Commons Attribution (CC BY) license (https:// creativecommons.org/licenses/by/ $4.0 /)$.

\begin{abstract}
In this study, we assessed the change in skin microbiota composition, relative abundance, and diversity with skin physiology disruption induced by SLS patch. Healthy women declaring to have a reactive skin were submitted to a $0.5 \%$ aqueous sodium lauryl sulfate solution application under occlusive patch condition for $24 \mathrm{~h}$. Skin properties were characterized by tewametry, corneometry, and colorimetry and bacterial diversity was assessed by $16 \mathrm{~S}$ rRNA sequencing. Analysis before and one day after SLS patch removal revealed an increase of skin redness and a decrease of stratum corneum hydration and skin barrier function. The relative abundance of taxa containing potential pathogens increase (Firmicutes: Staphylococcaceae; Proteobacteria: Enterobacteriaceae, Pantoea) while some of the most occurring Actinobacteria with valuable skin protection and repair capacities decreased (Micrococcus, Kocuria, and Corynebacterium). We observed an impaired skin barrier function and dehydration induced by SLS patch disturb the subtle balance of skin microbiota towards skin bacterial community dysbiosis. This study provides new insights on the skin bacterial composition and skin physiology simultaneously impaired by a SLS patch.
\end{abstract}

Keywords: SLS patch; skin barrier; dysbiosis; skin microbiota

\section{Introduction}

The skin, commonly described as the largest organ of our body, is an effective barrier to external environment. Thus, skin acts as a physical barrier that shields our body from the harmful effects of solar radiation, pathogenic germs and toxic substances. The skin locks water within itself to provide a proper moisturization which is mandatory for its homeostasis. This protective role is not only provided by the physical barrier aspect of the skin. Indeed, just like other epithelia, skin is colonized by an abundant and diverse community of microorganisms that collectively have been referred to as the "microbiota" [1]. It is now established that skin and other epithelia are more than passive hosts of this flora; skin interacts extensively with skin microbiota members to protect the body against infection from external pathogens. Skin microbiota are composed of bacteria, viruses, and fungi with differing distribution according to the environmental niche they colonize, e.g., dry, moist, or sebaceous [2,3]. These microorganisms can be categorized as resident or transient with different modes of interaction with the skin: commensal, mutualistic, neutral or pathogenic. Among skin microbiota members, bacteria residing across all skin fall into four phyla: Actinobacteria (52\%) represented by the genera Cutibacterium formerly known as Propionibacterium, Micrococcus, Corynebacteria; Firmicutes (24\%) represented by the genera Staphylococcus, Lactobacillus, and Streptococcus; Proteobacteria (16\%) that 
include the genera Paracoccus and Sphingomonas, and Bacteroidetes $(5 \%)$ including the genera Prevotella and Porphyromonas [4,5]. Resident bacteria are generally described as commensal. However, there is more and more evidence that they are mutualistic to skin cells, meaning that skin and microbiota mutually benefit from their cohabitation [6]. Commensal microorganisms have been shown to be beneficial for skin defense and health. In addition, researches related to skin microbiome showed that a balanced resident skin microbiota is one of the requirements towards a healthy skin $[1,5,7,8]$.

Our skin is subjected daily to topical care products, like hygiene products, which are used to remove contaminants on its surface and prevent infection. Although concerns have been expressed about the possible effects of detergent contained in hygiene and cosmetic product on the skin integrity and function [9], very few investigations have been conducted to show how they are impacting the skin barrier function and skin microbiota composition at the same time. Most of the existing literature and the prevailing understanding of hygiene in general are based on cultivation method with limited inputs and no taxonomic information.

Sodium lauryl sulfate (SLS) is a widely used surfactant in cleansing products, cosmetics at dose ranging from 0.01 to $50 \%$ [10] and personal care products [11,12]. SLS is an anionic surfactant which distorts cell membrane proteins [13]. It is also known to induce irritation under occlusive patch application starting at $0.5 \%[14,15]$ and is regularly used in this way in cosmetic studies. In order to better understand its effect in the context of the occlusive patch, we conducted a study to assess the effect of SLS applied as a patch on skin physiology and skin bacterial community. We used $16 \mathrm{~S}$ metabarcoding technology for a deeper exploration on skin bacterial community composition, relative abundance, and diversity after SLS patch application. This monocentric, open before-after study was conducted on healthy adult French women declaring to have a reactive skin. Skin physiology through transepidermal water loss (TEWL), hydration of the stratum corneum, skin color, as well as skin bacterial community composition were compared before and after SLS patch application on the back.

\section{Materials and Methods}

\subsection{Study Design}

All study volunteers were fully informed of the study protocol and informed consent was signed prior to inclusion of study participants. This project was operationally carried out in Biofortis facility (location authorized by the French regional health agency) before the application of the Jardé law and was therefore not qualified as having to be approved by an external ethic committee.

Thirty healthy Caucasian female volunteers with a fair skin (Fitzpatrick skin type II or III), aged 18 to 45 , with body mass index (BMI) between 18.5 and $29.9 \mathrm{~kg} \mathrm{~m}^{-2}$, and declaring to have a reactive skin, were recruited in 3 months (December 2016 to February 2017) from one French site (Biofortis Mérieux NutriSciences, a Contract research organization, based in Saint-Herblain, France). Women were excluded if they were menopaused; had an active skin disease on the tested areas, or had scars, tattoos, moles or another patch in the back; were allergic to cosmetics; or if they suffered from a severe chronic disease, infectious disease, metabolic disorder, or any other pathology able to disrupt skin health (eczema, psoriasis, seborrheic dermatitis, ...). Exclusion criteria also included antibiotic, antifungal, immunosuppressive, anti-histaminic or corticosteroid treatments during the 2 months preceding the study; pre/probiotics treatments during the month preceding the study; exposition to the sun/UV radiations or participation to a similar cosmetic study during the previous month of the study.

The design of the study included 3 visits: $\mathrm{V} 0$ as a baseline for skin physiology/microbiota assessment and SLS patch application, V1 for patch removal, V2 for skin physiology/microbiota assessment. The surfactant SLS (Sigma Aldrich, Saint-Quentin-Fallavier, France) was applied on a $20 \mathrm{~cm}^{2}$ skin area on the upper back as a patch during visit V0. Two sites approximately 20 $\mathrm{cm}^{2}$ each were selected on each side of the back. On each site, an aqueous $0.5 \%$ SLS solution 
was applied on a compress by a nurse to the pre-marked test areas under occlusive adhesive plaster. After $24 \mathrm{~h}$ of SLS patch application, the subjects came back to the study center and the SLS patch was removed (V1). Volunteers came back again one day after patch removal (V2) for the assessment of SLS patch effect on skin physiology through clinical and bacterial community measurement and analysis.

Forty-eight hours before the inclusion visit and during the study, the subjects were prevented from peeling in the back, bathing and/or swimming in pool or sea. Sauna and/or hammam, physical exercise involving high sweating, change of life habits (quality of life, diet, tobacco...), as well as change or start of concomitant treatments were also excluded. Each morning visit (at V0, V1, and V2), neither washing procedure nor care product was allowed in the back.

\subsection{Skin Clinical Evaluation, Sample Collection and Analysis}

Before instrumental measurement and skin bacteria sampling during the visits, subjects remained relaxed and must have had a rest period of 5-10 $\mathrm{min}$ in an acclimatized room (temperature: $21 \pm 1{ }^{\circ} \mathrm{C}$, humidity: 50\% $\pm 10 \%$ ). The subjects were lying down, back unclothed. The measurements were made on the same challenged skin area (both sides). The endpoints were also measured at baseline and $24 \mathrm{~h}$ after patch removal, thanks to Löffler and Happle's results showing a maximum skin barrier impairment $24 \mathrm{~h}$ after patch removal.

\subsection{Clinical Evaluation}

Transepidermal Water Loss (TEWL) was measured by a noninvasive and sensitive method using tewametry (MPA TewameterVR TM300, Courage \& Khazaka, Köln, Germany) to quantify stratum corneum barrier function $[16,17]$. For this skin parameter, the probe was put on the skin continuously until the value reached a stability zone which corresponded to an average value reported by the technician as the TEWL score. There was one measurement per test area per assessment time.

Skin hydration was determined by corneometry (Corneometer CM825, Courage \& Khazaka, Germany) which measures the electrical capacitance of the skin surface. The latter is considered a function of the water content in the stratum corneum [18]. Three measurements were performed in the test areas per assessment time.

Skin aspect namely color and luminosity were assessed using a Colorimeter (Skin Colorimer CL 400, Courage \& Khazaka, Germany) which provides an accurate measurement of color via a * parameter (intensity of red versus green), and of brightness via the $L^{*}$ parameter (indicating the lightness/darkness).

\subsection{Skin Bacterial $16 S$ rRNA Gene Metabarcoding}

Skin microbiome was sampled by swabbing an area of $20 \mathrm{~cm}^{2}\left(4 \times 5 \mathrm{~cm}^{2}\right)$ of each side on the back (Copan cotton Swab, VWR, Chevillon-sur-Huillard, France). The location was determined bya cutaneous marking on the site during the baseline visit. Reproducibility of the sampling was reached using a known sterile plastic surface placed on the skin of the volunteers. Swabs were then submitted to DNA extraction using a mechanical lysis by bead beating (FastPrep-24, MPBiomedicals, Santa Ana, CA, USA), and a chemical lysis (Tris-SDS) followed by phenol-chloroform DNA isolation. Resulting DNA solutions in Tris-EDTA were then quantified by fluorimetry (Qubit 2.0 Fluorometer).

Preparation of 16S rRNA gene amplicon libraries, sequencing, and data processing:

PCR amplification was performed using $16 \mathrm{~S}$ universal primers 341F and 785R [19] targeting the variable V3-V4 region of the bacterial 16S ribosomal gene. The 16S V3-V4 amplicon size was verified by capillary electrophoresis (2100 BioAnalyzer, Agilent Technologies, Santa Clara, CA, USA) onto at least $10 \%$ of the samples. All of them were purified with magnetic beads (AMPure XP beads, Beckman Coulter France, Villepinte, France).

Then, for each sample, a sequencing library was generated by addition of dual indices and Illumina sequencing adapters using Nextera XT Index kit. Each library was cleaned 
up with magnetic beads and the size verified by capillary electrophoresis onto at least $10 \%$ of the samples. After quantification by fluorimetry (Qubit 2.0 Fluorometer), libraries were normalized and pooled before denaturing and sequencing using the Illumina MiSeq platform using $2 \times 250$ paired-end Miseq kit V2. Multiplexing was conducted in order to theoretically reach 50,000 raw reads per sample.

The targeted metagenomic sequences from skin microbiota were analyzed using an in-house bioinformatics pipeline based on mothur software [20]. Basically, single read sequences were paired for each sample into longer fragments and cleaned. Sequences were trimmed and aligned to the V3-V4 region of the 16S rDNA gene of the Greengenes database formatted by mothur (gg_13_5_99 release). Chimeric sequences were eliminated using the UCHIME algorithm [21]. Filtered reads were then classified using naïve Bayesian classifier [22] against the Ribosomal Database Project (RDP) 16S rDNA gene training set v11 with a bootstrap cutoff of $60 \%$. Sequences were then clustered into operational taxonomic units (OTUs) using furthest-neighbour clustering at a similarity threshold of $97 \%$. Note that the RDP version used in this study retains name Propionibacterium now renamed Cutibacterium.

Outlier taxonomic profiles identified by quality control (QC) analysis defaults or non-biological consistence with skin flora which may be due to sampling processes were excluded from statistical analysis.

Relative abundances of taxa at the phylum, family and genus classification levels were produced and graphical representations, such as bar plots, were made based on these relative abundances (heatmaps are presented as supplementary data). To improve the readability of graphics, taxa present in average in all samples at a threshold $\geq 0.5 \%$ or present in at least $10 \%$ of samples at a threshold $\geq 0.5 \%$ were individually represented. In other cases, taxa were grouped and labelled "other". In addition, taxa present at a threshold $\leq 0.01 \%$ were not represented in heatmaps due to the logarithmic colorimetric scale used.

For alpha diversity, the abundance-based coverage estimator (ACE), the Shannon index, the Fisher's alpha parameter, and the inverse Simpson index were calculated for each sample. The ACE index gives an estimation of the real richness of the skin while the Shannon index, the Fisher's alpha parameter and the inverse Simpson index are diversity estimators. The Shannon index integrates both richness and evenness and gives an idea of the equitability profile of the population. The Fisher's alpha parameter corresponds to the number of new OTUs that could be discovered by increasing the sampling effort of $1 \log$. The inverse Simpson can be seen as the number of dominant species, equivalent to OTU in this study.

\subsection{Statistical Analysis}

Statistical analyzes and graphical representations of clinical data were performed using SAS software version 9.3 (SAS Institute Inc., Cary, NC, USA). Graphical representations of microbiome data were generated using $\mathrm{R}$ software version 3.2.3. For all statistical tests (two-sided), the 0.05 level of significance was used to justify a claim of a statistically significant effect.

Descriptive results are presented as mean \pm standard deviation (SD). Study endpoints (alpha-diversity indices, relative abundances of taxa, TEWL, skin color and corneometry measurements) were compared before and 1 day after the SLS patch removal (V0 vs. V2) by using an analysis of variance (ANOVA) model for repeated measurements (SAS PROC MIXED), taking into account data correlation between measurements from the same subject (two sides on the back and two visits). Estimated averages are reported with $95 \%$ confidence interval $(95 \% \mathrm{CI})$. At each taxonomic level, a Benjamini Hochberg procedure was applied to control the false discovery rate due to multiple hypothesis tests on all taxa (adjusted $p$-values are reported). 


\section{Results}

\subsection{Clinical Evaluation}

The skin physiology analyzes were carried out on the group of 29 volunteers who completed the study and who made up a population with an average age of $32.0 \pm 6.4$ years (from 21 to 44 years) for TEWL and skin color and capacitance measurement.

\section{Transepidermal Water Loss}

An increase in TEWL reflects impairment in skin barrier function. The mean TEWL value was $5.1 \pm 2.3 \mathrm{~g} \mathrm{~m}^{-2} \mathrm{~h}^{-1}$ at baseline (comparable on both sides of the back of each volunteer) while $42.6 \pm 6.8 \mathrm{~g} \mathrm{~m}^{-2} \mathrm{~h}^{-1}$ one day after the SLS patch removal, resulting in a statistically significant increase of $37.5 \mathrm{~g} \mathrm{~m}^{-2} \mathrm{~h}^{-1}$ [95\% CI: 36.1 to 39.0] $(p<0.0001)$ reflecting a marked and significant skin barrier function disruption induced by the SLS patch (Table 1).

Table 1. Change in transepidermal water loss, stratum corneum hydration and skin color from baseline (V0) to the day following SLS patch removal (V2).

\begin{tabular}{|c|c|c|c|c|}
\hline & $\begin{array}{c}\text { Baseline(V0) } \\
\text { Mean (SD) }\end{array}$ & $\begin{array}{c}24 \mathrm{~h} \text { after SLS Patch } \\
\text { Removal (V2) } \\
\text { Mean (SD) }\end{array}$ & $\begin{array}{c}\text { Estimated Mean } \\
\text { Change (V2-V0) } \\
{[95 \% \mathrm{CI}]}\end{array}$ & $p$-Value \\
\hline TEWL ${ }^{1}$ score $\left(\mathrm{g} \mathrm{m}^{-2} \mathrm{~h}^{-1}\right)$ & $5.1(2.28)$ & $42.6(6.79)$ & 37.5 [36.1 to 39.0] & $<0.0001$ \\
\hline Colorimetry score $\mathrm{L}^{*}$ (a.u. ${ }^{2}$ ) & $65.3(2.98)$ & $60.7(2.92)$ & $-4.7[5.2$ to 4.1$]$ & $<0.0001$ \\
\hline Colorimetry score $\mathrm{a}^{*}$ (a.u.) & $10.7(1.61)$ & $17.5(1.86)$ & $6.8[6.4$ to 7.2$]$ & $<0.0001$ \\
\hline Corneometry score (a.u.) & $45.1(8.19)$ & $39.7(10.70)$ & $-5.4[-8.0$ to -2.8$]$ & $<0.0001$ \\
\hline
\end{tabular}

${ }^{1}$ TEWL: Transepidermal water loss; ${ }^{2}$ a.u. arbitrary unit.

\subsection{Skin Color, Luminosity and Moisturization}

The three studied skin parameters, skin brightness, redness and stratum corneum hydration were comparable at baseline on both side of the back of each volunteer, and significantly changed after the SLS patch removal (Table 1).

The average Colorimeter score $\mathrm{L}^{*}$ related to brightness was $65.3 \pm 3.0$ at baseline while $60.7 \pm 3.0$ one day after the SLS patch removal, resulting in a statistically significant decrease of -4.7 [95\% CI: -5.2 to -4.1$](p<0.0001)$.

The average Colorimeter score $a^{*}$ was $10.7 \pm 1.6$ at baseline while $17.5 \pm 1.9$ one day after the SLS patch removal, resulting in a statistically significant increase of 6.8 units [95\% CI: 6.4 to 7.2] $(p<0.0001)$ evidencing thus increase in skin redness.

For skin moisturization, values less than 30 a.u. represent very dry skin, 30-40 a.u. reflect dry skin, and values superior to 40 a.u. are typically associated with normal skin. Thus, a decrease in Corneometer values corresponds to skin drying effect [23].

The average corneometry score was $45.1 \pm 8.2$ at baseline while $39.7 \pm 10.7$ one day after the SLS patch removal, resulting in a statistically significant decrease of -5.4 units [95\% CI: -8.0 to -2.8$](p<0.0001)$. At baseline, the skin areas designated to be treated had an average Corneometer value corresponding to normal skin. The electrical capacitance significantly decreased $24 \mathrm{~h}$ after SLS patch removal (Table 1 ).

\subsection{Skin Microbiota Analysis}

Skin bacterial community data were available for 27 women at baseline (one side of the back unavailable for one woman and one volunteer did not pass quality control at the DNA extraction step). Further, 24 women's skin bacteria data were available one day after the SLS patch removal (five did not pass quality control at the sequencing step). Alpha diversity indices are presented in Figure 1. 

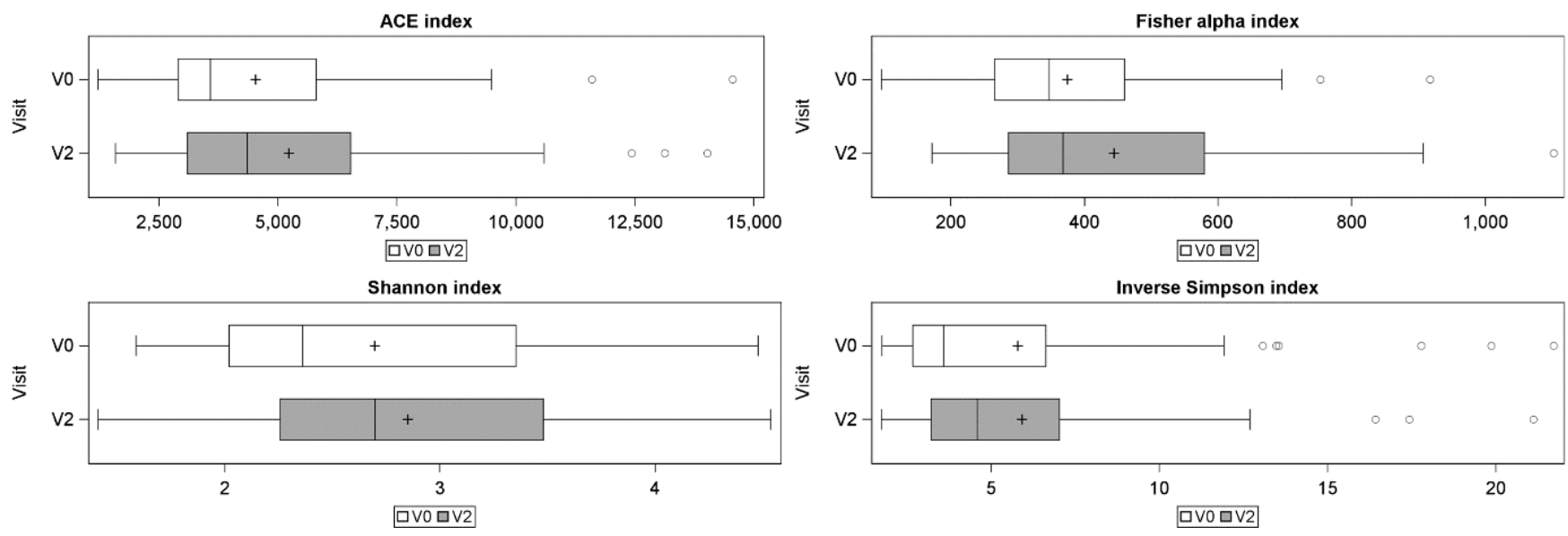

Figure 1. Alpha diversity indices before (V0) and one day after (V2) SLS patch removal.

The average ACE index was significantly higher after SLS patch (750.8 [95\% CI: 115.7 to 1385.9]; $p=0.0211$ ) evidencing an increase in richness after SLS patch. The average Fisher alpha index was significantly higher after SLS (76.3 [95\% CI: 26.3 to 126.3]; $p=0.0032$ ) evidencing the increase in richness and more specifically the presence of additional bacterial species at a low relative abundance after SLS patch.

This increase of richness conducted to a higher overall diversity as confirmed by the Shannon diversity index which was significantly higher after SLS patch application $(0.25$ [95\% CI: 0.11 to 0.39 ]; $p=0.0005)$. Finally, there was no statistically significant differences for the Inverse Simpson index after the SLS patch (0.59 [95\% CI: -0.56 to 1.75]; $p=0.3097)$, indicating, on one hand, that the cutaneous flora was still dominated by the same small number of dominant species, and confirming on the other hand that the variation concerned sub-dominant bacterial species.

Change in skin bacteria community structure was also analyzed. At baseline as reported in literature more than $90 \%$ of resident taxa belonged to Actinobacteria (mean relative abundance of $68.63 \% \pm 24.84 \%)$, Firmicutes $(19.69 \% \pm 23.55 \%)$ and Proteobacteria $(11.06 \% \pm 13.66 \%)$ phyla, with preponderance in Propionibacterium (Cutibacterium), Staphylococcus, Paracoccus, Enhydrobacter, Micrococcus, and Corynebacterium genera. After SLS patch, there was significant shifts of skin bacterial community composition at different taxonomic levels (Figure 2, Supplementary Figures S1 and S2).

The average relative abundance of Actinobacteria (Figure 2A, Figures S1A and S2A), the major phylum of skin microbiota, were significantly lower after SLS patch compared to baseline (absolute mean change: $-9.67 \%$ [95\%CI: $-14.93 \%$ to $-4.40 \%], p=0.0024$ ). This variation has also been observed at lower taxonomic levels (Figure 2B,C and Supplementary Figures S1B,C and S2B,C).

The average relative abundance of Micrococcaceae family significantly decreased after SLS patch: from $4.67 \%$ to $3.07 \%$ (absolute average change of $-1.59 \%$ [95\%CI: -2.50 to $-0.68], p=0.0310)$ respectively. The same trend has been evidenced with other family belonging to Actinobacteria like Dermacoccaceae, Propionibacteriaceae, Brevibacteriaceae, Dermabacteraceae, and Corynebacteriaceae, despite the fact that statistical significance was not reached (adjusted $p$-value between 0.05 and 0.10 ). In addition, at the genus level, the average relative abundance of Micrococcus (Micrococcaceae, Actinobacteria) and Kocuria (Micrococcaceae, Actinobacteria) was also lower after SLS patch (absolute mean change of $-1.15 \%$ [ $95 \% \mathrm{CI}:-1.89 \%$ to $-0.41 \%$ ], $p=0.0485$; and $-0.59 \%$ [95\%CI: $-0.89 \%$ to $-0.29 \%$ ], $p=0.0111$ respectively).

At the same time, the average relative abundance of Firmicutes phylum significantly increased after SLS: from $19.02 \%$ to $26.11 \%$ (absolute average change of $7.09 \%$ [95\%CI: $2.83 \%$ to $11.35 \%, p=0.0035$ ). At lower level, a trend in increase was observed for Staphylococcaceae belonging to Firmicutes (absolute mean change of $6.55 \%$ [95\%CI: $2.10 \%$ to $10.99 \%], p=0.0579$ ). 

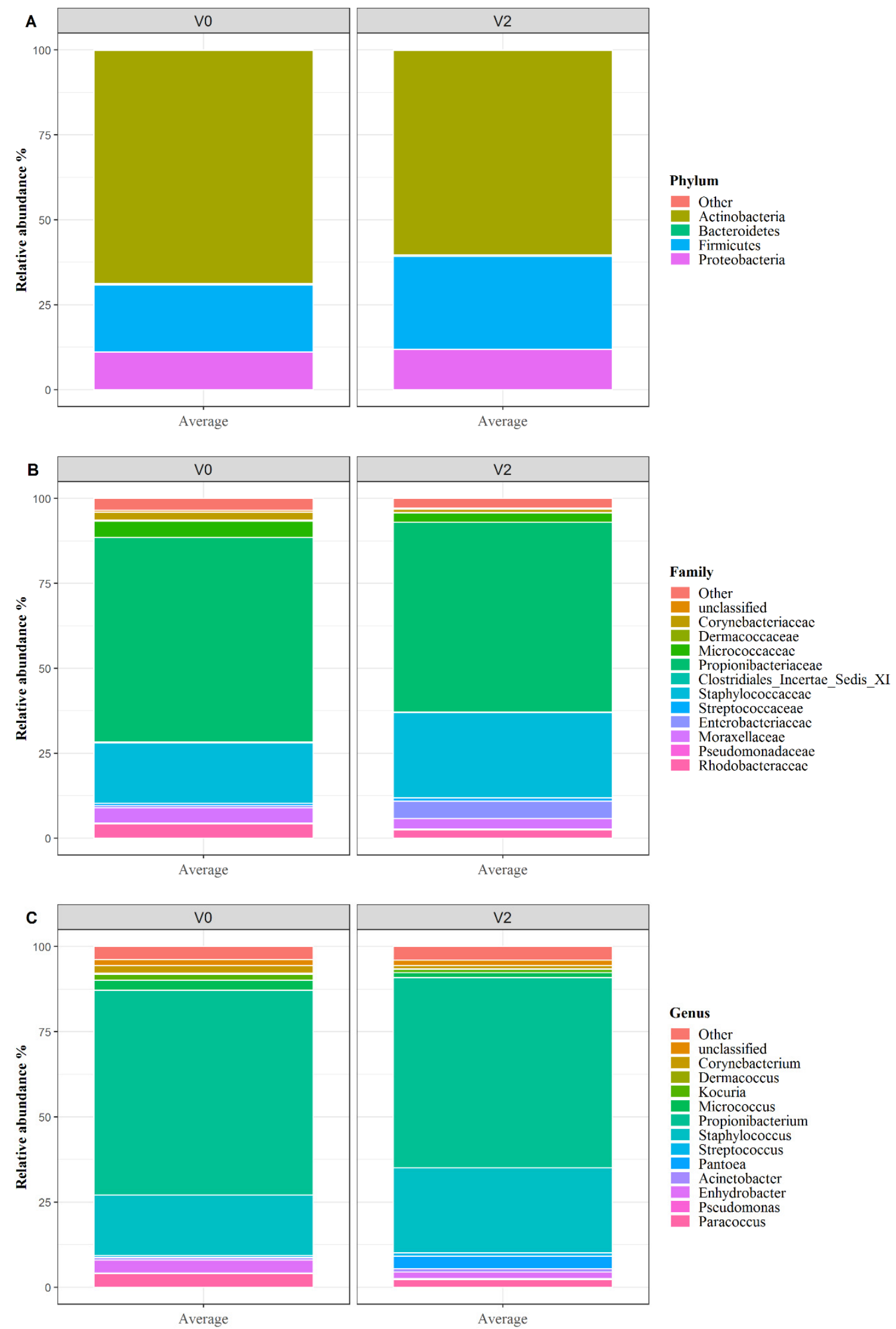

Figure 2. Taxonomic composition of the skin flora by 16S sequencing before and after SLS patch application and removal at different level of classification. (A) Phylum level; (B) family level and (C) genus level. 
After the SLS patch, Proteobacteria phylum composition was significantly disturbed for one family and one genus: the Rhodobacteriaceae family and the Paracoccus genus were significantly lower after SLS (absolute mean change of $-1.55 \%$ [95\%CI: $-2.47 \%$ to $-0.62 \%$, $p=0.0310$; and $-1.44 \%$ [95\%CI: $-2.23 \%$ to $-0.66 \%$ ] $p=0.0127$ respectively). Finally, the relative abundance of Enterobacteriaceae family and Pantoea genus directionally increased after SLS patch (absolute mean change of $4.74 \%$ [95\%CI: $1.18 \%$ to $8.30 \%$ ], $p=0.0579$ and $3.73 \%$ [95\%CI: $0.70 \%$ to $6.76 \%$ ], $p=0.0824$, respectively), with this change being especially marked for some subjects (Supplementary Figure S1B (purple color) and Figure S1C (blue color), subjects on left hand side).

\section{Discussion}

SLS is a surfactant used as a cleansing agent in common skincare products [11,12] and skin contact with detergent like SLS occurs on a daily basis. SLS as well as other synthetic surfactant can disturb skin lipids composition, remove lipids from stratum corneum surface and thereby disrupt the skin barrier function [24,25]. We have confirmed in this study, as already shown by other authors [26,27], that SLS applied as a patch induced skin barrier dysfunction by increasing TEWL and thus subsequently decreasing natural moisturizing factor, NMF, in skin moisturization: TEWL increase was significantly higher one day after SLS patch removal whereas skin moisturization was decreased. As expected, SLS patch also induced an increase in skin redness. Moreover, we evidenced for the first time a decrease in skin luminosity after a SLS patch. SLS patch effect on skin barrier and skin irritation is well documented. However, there are limited knowledge on the effect on skin bacterial community coming with this skin physiology impairment. Regarding the effect of some hygiene products on skin bacterial community, namely cleansers used in hospital, some authors have reported that acute use of cleanser can decrease antimicrobial peptides synthesis by the skin [28], while others pointed out that frequent washing hand procedures and the use of some cosmetics lead to changes in skin bacterial community [29,30] but these later studies gave limited information on the effects of such products on skin microbiota composition and ecology as they used culture method. Staudinger and coworkers have shown using $16 \mathrm{~S}$ sequencing that the use of make-up appeared to interfere with the composition of bacterial community, but this experience was conducted on a very small sample size and needs further confirmation [31].

We sought to explore the effects of SLS patch used to disrupt skin physiology on the skin bacterial communities by analyzing $16 \mathrm{~S}$ rRNA gene sequences from skin swabs sampled before and after SLS patch removal. Skin microbiota baseline analysis results were consistent to reported data [4]: upper back skin was dominated by Cutibacterium formerly named Propionibacterium followed by Staphylococcus. Back skin is an oily site like the forehead which normally has low diversity compared to other, less oily parts, like the forearm [32,33]. Mukherjee and coworkers showed that the diversity and richness of the skin microbiota decreased with increase in oiliness of the cheek [34] confirming observation of Li and coworkers. After SLS patch and skin barrier disruption, variations of several alpha-diversity indices evidencing an increase of richness and a limited modification of evenness, which means that in addition to appearance of new bacterial taxa, the skin bacterial keeps a population structure dominated by limited number of genera

Regarding the taxonomical composition of skin bacterial community, significant changes were observed at the same time than skin barrier disruption.

The relative abundance of Firmicutes phylum and the Staphylococcaceae family increased significantly after SLS patch. This increase in Staphylococcaceae can be linked to increase of resident commensal species like S. epidermidis or transient opportunistic pathogenic like $S$. aureus or both. Indeed, S. epidermidis is known to secrete antimicrobial peptides that kills some transient opportunistic pathogen like $S$. aureus [35]. It has antiinflammatory actions and promotes skin physical barrier function repair. For S. aureus, the disruption of skin barrier function with increase in TEWL and a decrease in skin moisturization makes a favorable environment for its growth $[29,36]$. We have also seen the rise in 
relative abundance of some potential pathogenic like the genus Pantoea (Proteobacteria, Enterobacteriaceae). Species belonging to Pantoea gram negative genus are known to be more environmental than resident to human skin [37]. Pantoea agglomerans has been isolated from lesional skin of children with atopic dermatitis [38] which is positively correlated with impaired skin barrier function.

Overall, SLS patch induced decrease in major resident commensal genera of different Phyla like Cutibacterium (Propionibacterium $p=0.082$ ), Micrococcus $(p=0.048)$, Corynebacterium $(p=0.056)$, Kocuria $(p=0.011)$, Dermacoccus $(p=0.061)$ and Paracoccus $(p=0.0127)$. These bacteria as the majority of resident commensal participate to skin protection and for some of them to skin repair. P. acnes (Cutibacterium acnes), which is described as a player in skin acne, provides protection to skin against other opportunistic pathogen by acidifying the skin [39]. These reductions in relative abundance were significant for Micrococcus, Kocuria and Corynebacterium, members of the most occurring Phylum of Actinobacteria with valuable capacities: Micrococcus luteus (Micrococacceae, Actinobacteria) has protective effect against UV [40]; it is known for producing an enzyme that can repair DNA damages induced by UV [41]. Kocuria genus which is resistant to UV are known to produce antioxidant enzyme such Superoxide dismutase [42]; we cannot exclude that these properties of Kocuria and Micrococcus are beneficial to skin protection and repair after different stresses like UV stress. More interestingly in Corynebacterium genus, another skin resident Corynebacterium striatum (Corynebacteriaceae, Actinobacteria) has been shown to shift opportunistic pathogens to commensalism by suppressing the expression of virulence related genes [43].

The decrease in lipohilic genera, like Propionibacterium, Micrococcus, and Corynebacterium, can be linked to the impairment or removal of skin lipids from the skin surface by SLS patch [44]. Paracoccus (Rhodobacteriaceae, Proteobacteria) depletion after SLS patch can also be detrimental as some useful and interesting properties have been described for this genus like its capacity of depollution on polycyclic aromatic hydrocarbons (PAH) which also deposit on skin [45].

The decrease in some skin commensal bacteria members can be attributed to disturbance induced by SLS patch on skin physical and chemical composition (lipids, moisturization) as well as potential SLS cytotoxicity toward skin bacterial community member. In this study we applied SLS using an occlusive patch to induce skin irritation and skin barrier disruption. This would have potentially created a temporary humid environment comparable to dressing or occlusion that could disturb skin bacteria composition and would have increased population of bacteria taxa living in humid environment like the armpit. Leow and Maibach reported an increase of TEWL as well as Gram-negative bacteria (that could be linked to the observed increase of Pantoea within our study) in a review on the occlusion effect on skin and our study showed an additional effect of SLS on other genera belonging to Actinobacteria, the most abundant skin bacteria phylum beside the occlusion [46]. SLS patch disturbs the skin physiology but also the subtle balance of the skin microbiota bacterial community, and this can may be detrimental for skin health and beauty. The skin bacterial community, through their interaction with the host's skin, protects the skin from some aggressions and helps to repair it when damaged.

\section{Conclusions}

Today, in Western countries, there is an increase in allergies and some skin conditions like dermatitis [47], including atopic dermatitis, eczema, or irritant contact dermatitis [48], that can be linked to skin microbiota variations. For these dermatological conditions, hygiene habits and particularly the intensive and frequent use of some harsh detergent account for their appearing $[23,49,50]$. Redefining new hygiene habits, with low detergent concentration and choosing mild surfactants with limited impact on the skin integrity, physiology, and bacterial community microbiome could be helpful to maintain the skin integrity and microbiota balance for a beneficial interaction with the skin host. 
Supplementary Materials: The following are available online at https:/ / www.mdpi.com/2079-9 284/8/1/6/s1, Figure S1: Taxonomic composition of the skin flora by $16 \mathrm{~S}$ sequencing at baseline (V0) and the day following SLS patch removal (V2). Barplots of individual relative abundances: A at Phylum level; B at family level and C at genus level., Figure S2: Taxonomic composition of the skin flora by $16 \mathrm{~S}$ sequencing at baseline (V0) and the day following SLS patch removal (V2). Heatmaps of individual relative abundances: A at Phylum level; B at family level and $\mathrm{C}$ at genus level.

Author Contributions: Conceptualization, S.L.F.-L., T.C., A.D.M., P.M., S.L.-O., and V.A.; methodology, S.L.F.-L., T.C., and C.B.-R.; formal analysis, S.L.F.-L., T.C., C.B.-R., P.M., and S.L.-O.; investigation, T.C., C.B.-R., and S.L.-O.; statistics, F.G., S.L., and B.D.; writing-original draft preparation, T.C. and S.L.-O.; writing-review \& editing, F.G., S.L., and S.L.-O.; visualization, B.D.; project administration, S.L.F.-L., T.C., and S.L.-O. All authors have read and agreed to the published version of the manuscript.

Funding: This research received no external funding.

Institutional Review Board Statement: This project was operationally carried out in Biofortis facility (location authorized by the french regional health agency). Ethical review and approval have been done internally. Indeed, this study was conducted before the application of the Jardé law and was therefore not qualified as having to be approved by an external ethics committee.

Informed Consent Statement: Informed consent was obtained from all subjects involved in the study.

Data Availability Statement: Not applicable.

Conflicts of Interest: The authors declare no conflict of interest.

\section{References}

1. Schommer, N.N.; Gallo, R.L. Structure and function of the human skin microbiome. Trends Microbiol. 2013, 21, 660-668. [CrossRef] [PubMed]

2. Grice, E.A.; Segre, J.A. The skin microbiome. Nat. Rev. Microbiol. 2011, 9, 244-253. [CrossRef] [PubMed]

3. Perez Perez, G.I.; Gao, Z.; Jourdain, R.; Ramirez, J.; Gany, F.; Clavaud, C.; Demaude, J.; Breton, L.; Blaser, M.J. Body Site Is a More Determinant Factor than Human Population Diversity in the Healthy Skin Microbiome. PLoS ONE 2016, 11, e0151990. [CrossRef] [PubMed]

4. Cundell, A.M. Microbial Ecology of the Human Skin. Microb. Ecol. 2018, 76, 113-120. [CrossRef] [PubMed]

5. Grice, E.A.; Kong, H.H.; Conlan, S.; Deming, C.B.; Davis, J.; Young, A.C.; NISC Comparative Sequencing Program; Bouffard, G.G.; Blakesley, R.W.; Murray, P.R.; et al. Topographical and temporal diversity of the human skin microbiome. Science 2009, 324, 1190-1192. [CrossRef]

6. Vandegrift, R.; Bateman, A.C.; Siemens, K.N.; Nguyen, M.; Wilson, H.E.; Green, J.L.; Van Den Wymelenberg, K.G.; Hickey, R.J. Cleanliness in context: Reconciling hygiene with a modern microbial perspective. Microbiome 2017, 5, 76. [CrossRef]

7. Branco, C.T.; Guimaraes, J.P. Modulation of skin microbiota by topical prebiotics. TKS Publ. 2015, 10, 21-27.

8. Sanford, J.A.; Gallo, R.L. Functions of the skin microbiota in health and disease. Semin. Immunol. 2013, 25, 370-377. [CrossRef]

9. Draelos, Z.D. The science behind skin care: Cleansers. J. Cosmet. Dermatol. 2018, 17, 8-14. [CrossRef]

10. Bondi, C.A.; Marks, J.L.; Wroblewski, L.B.; Raatikainen, H.S.; Lenox, S.R.; Gebhardt, K.E. Human and Environmental Toxicity of Sodium Lauryl Sulfate (SLS): Evidence for Safe Use in Household Cleaning Products. Environ. Health Insights 2015, 9, 27-32. [CrossRef]

11. Fiume, M.; Bergfeld, W.F.; Belsito, D.V.; Klaassen, C.D.; Marks, J.G.; Shank, R.C.; Slaga, T.J.; Snyder, P.W.; Alan Andersen, F. Final report on the safety assessment of sodium cetearyl sulfate and related alkyl sulfates as used in cosmetics. Int. J. Toxicol. 2010, 29, 115S-132S. [CrossRef]

12. Larrouy, M. Le Sodium Laury Sulfate. 2015. Available online: https://www.scc-quebec.org/wp-content/uploads/2017/08/ Monographie-Sodium-Lauryl-Sulfate-Malaury-Larrouy-2015.pdf (accessed on 1 December 2015).

13. Piret, J.; Lamontagne, J.; Bestman-Smith, J.; Roy, S.; Gourde, P.; Désormeaux, A.; Omar, R.F.; Juhász, J.; Bergeron, M.G. In vitro and in vivo evaluations of sodium lauryl sulfate and dextran sulfate as microbicides against herpes simplex and human immunodeficiency viruses. J. Clin. Microbiol. 2000, 38, 110-119. [PubMed]

14. Gabard, B.; Chatelain, E.; Bieli, E.; Haas, S. Surfactant irritation: In vitro corneosurfametry and in vivo bioengineering. Skin Res. Technol. 2001, 7, 49-55. [CrossRef]

15. Lee, E.; Kim, S.; Lee, J.; Cho, S.-A.; Shin, K. Ethnic differences in objective and subjective skin irritation response: An international study. Skin Res. Technol. 2014, 20, 265-269. [CrossRef] [PubMed]

16. Antonov, D.; Schliemann, S.; Elsner, P. Methods for the Assessment of Barrier Function. Curr. Probl. Dermatol. 2016, 49, 61-70. [CrossRef] [PubMed]

17. Pinnagoda, J.; Tupker, R.A.; Agner, T.; Serup, J. Guidelines for transepidermal water loss (TEWL) measurement. A report from the Standardization Group of the European Society of Contact Dermatitis. Contact Dermat. 1990, 22, 164-178. [CrossRef] [PubMed] 
18. Clarys, P.; Clijsen, R.; Taeymans, J.; Barel, A.O. Hydration measurements of the stratum corneum: Comparison between the capacitance method (digital version of the Corneometer CM $825^{\circledR}$ ) and the impedance method (Skicon-200EX ${ }^{\circledR}$ ). Skin Res. Technol. 2012, 18, 316-323. [CrossRef]

19. Klindworth, A.; Pruesse, E.; Schweer, T.; Peplies, J.; Quast, C.; Horn, M.; Glöckner, F.O. Evaluation of general 16S ribosomal RNA gene PCR primers for classical and next-generation sequencing-based diversity studies. Nucleic Acids Res. 2013, 41, e1. [CrossRef]

20. Schloss, P.D.; Westcott, S.L.; Ryabin, T.; Hall, J.R.; Hartmann, M.; Hollister, E.B.; Lesniewski, R.A.; Oakley, B.B.; Parks, D.H.; Robinson, C.J.; et al. Introducing mothur: Open-Source, Platform-Independent, Community-Supported Software for Describing and Comparing Microbial Communities. Appl. Environ. Microbiol. 2009, 75, 7537-7541. [CrossRef]

21. Edgar, R.C.; Haas, B.J.; Clemente, J.C.; Quince, C.; Knight, R. UCHIME improves sensitivity and speed of chimera detection. Bioinform. Oxf. Engl. 2011, 27, 2194-2200. [CrossRef]

22. Wang, Q.; Garrity, G.M.; Tiedje, J.M.; Cole, J.R. Naïve Bayesian Classifier for Rapid Assignment of rRNA Sequences into the New Bacterial Taxonomy. Appl. Environ. Microbiol. 2007, 73, 5261-5267. [CrossRef] [PubMed]

23. Angelova-Fischer, I.; Dapic, I.; Hoek, A.-K.; Jakasa, I.; Fischer, T.W.; Zillikens, D.; Kezic, S. Skin barrier integrity and natural moisturising factor levels after cumulative dermal exposure to alkaline agents in atopic dermatitis. Acta. Derm. Venereol. 2014, 94, 640-644. [CrossRef] [PubMed]

24. Chen, Y.-C.; Wang, P.-R.; Lai, T.-J.; Lu, L.-H.; Dai, L.-W.; Wang, C.-H. Using therapeutic ultrasound to promote irritated skin recovery after surfactant-induced barrier disruption. Ultrasonics 2019, 91, 206-212. [CrossRef]

25. Törmä, H.; Lindberg, M.; Berne, B. Skin barrier disruption by sodium lauryl sulfate-exposure alters the expressions of involucrin, transglutaminase 1, profilaggrin, and kallikreins during the repair phase in human skin in vivo. J. Investig. Dermatol. 2008, 128, 1212-1219. [CrossRef]

26. Stettler, H.; Kurka, P.; Lunau, N.; Manger, C.; Böhling, A.; Bielfeldt, S.; Wilhelm, K.-P.; Dähnhardt-Pfeiffer, S.; Dähnhardt, D.; Brill, F.H.H.; et al. A new topical panthenol-containing emollient: Results from two randomized controlled studies assessing its skin moisturization and barrier restoration potential, and the effect on skin microflora. J. Dermatol. Treat. 2017, 28, 173-180. [CrossRef]

27. Wilhelm, K.P.; Freitag, G.; Wolff, H.H. Surfactant-induced skin irritation and skin repair: Evaluation of a cumulative human irritation model by noninvasive techniques. J. Am. Acad. Dermatol. 1994, 31, 981-987. [CrossRef]

28. Two, A.M.; Nakatsuji, T.; Kotol, P.F.; Arvanitidou, E.; Du-Thumm, L.; Hata, T.R.; Gallo, R.L. The Cutaneous Microbiome and Aspects of Skin Antimicrobial Defense System Resist Acute Treatment with Topical Skin Cleansers. J. Investig. Dermatol. 2016, 136, 1950-1954. [CrossRef] [PubMed]

29. Larson, E.L.; Hughes, C.A.; Pyrek, J.D.; Sparks, S.M.; Cagatay, E.U.; Bartkus, J.M. Changes in bacterial flora associated with skin damage on hands of health care personnel. Am. J. Infect. Control 1998, 26, 513-521. [CrossRef]

30. Rocha, L.A.; de Borges, L.F.D.A.; Gontijo Filho, P.P. Changes in hands microbiota associated with skin damage because of hand hygiene procedures on the health care workers. Am. J. Infect. Control 2009, 37, 155-159. [CrossRef]

31. Staudinger, T.; Pipal, A.; Redl, B. Molecular analysis of the prevalent microbiota of human male and female forehead skin compared to forearm skin and the influence of make-up: Skin microbiota of human forehead compared to forearm. J. Appl. Microbiol. 2011, 110, 1381-1389. [CrossRef]

32. Li, X.; Yuan, C.; Xing, L.; Humbert, P. Topographical diversity of common skin microflora and its association with skin environment type: An observational study in Chinese women. Sci. Rep. 2017, 7, 18046. [CrossRef]

33. Shibagaki, N.; Suda, W.; Clavaud, C.; Bastien, P.; Takayasu, L.; Iioka, E.; Kurokawa, R.; Yamashita, N.; Hattori, Y.; Shindo, C.; et al. Aging-related changes in the diversity of women's skin microbiomes associated with oral bacteria. Sci. Rep. 2017, 7, 10567. [CrossRef]

34. Mukherjee, S.; Mitra, R.; Maitra, A.; Gupta, S.; Kumaran, S.; Chakrabortty, A.; Majumder, P.P. Sebum and Hydration Levels in Specific Regions of Human Face Significantly Predict the Nature and Diversity of Facial Skin Microbiome. Sci. Rep. 2016, 6, 36062. [CrossRef] [PubMed]

35. Chen, Y.E.; Fischbach, M.A.; Belkaid, Y. Skin microbiota-host interactions. Nature 2018, 553, 427-436. [CrossRef] [PubMed]

36. Baldwin, H.E.; Bhatia, N.D.; Friedman, A.; Eng, R.M.; Seite, S. The Role of Cutaneous Microbiota Harmony in Maintaining a Functional Skin Barrier. J. Drugs Dermatol. JDD 2017, 16, 12-18. [CrossRef] [PubMed]

37. Dutkiewicz, J.; Mackiewicz, B.; Kinga Lemieszek, M.; Golec, M.; Milanowski, J. Pantoea agglomerans: A mysterious bacterium of evil and good. Part III. Deleterious effects: Infections of humans, animals and plants. Ann. Agric. Environ. Med. AAEM 2016, 23, 197-205. [CrossRef] [PubMed]

38. Bilal, J.A.; Ahmad, M.I.; Robaee, A.A.A.; Alzolibani, A.A.; Shobaili, H.A.A.; Al-Khowailed, M.S. Pattern of bacterial colonization of atopic dermatitis in saudi children. J. Clin. Diagn. Res. JCDR 2013, 7, 1968-1970. [CrossRef]

39. Christensen, G.J.M.; Brüggemann, H. Bacterial skin commensals and their role as host guardians. Benef. Microbes 2014, 5, 201-215. [CrossRef]

40. Leung, M.H.Y.; Tong, X.; Bastien, P.; Guinot, F.; Tenenhaus, A.; Appenzeller, B.M.R.; Betts, R.J.; Mezzache, S.; Li, J.; Bourokba, N.; et al. Changes of the human skin microbiota upon chronic exposure to polycyclic aromatic hydrocarbon pollutants. Microbiome 2020, 8, 100. [CrossRef]

41. Basílico, G.; Roger, C.A.; Seigelchifer, M.; Kerner, N. UV-specific DNA repair recombinant fusion enzyme: A new stable pharmacologically active principle suitable for photoprotection. J. Dermatol. Sci. 2005, 39, 81-88. [CrossRef] 
42. Shukla, M.R.; Yadav, R.; Desai, A. Catalase and superoxide dismutase double staining zymogram technique for Deinococcus and Kocuria species exposed to multiple stresses: Catalase and superoxide dismutase double staining zymogram technique for Deinococcus and Kocuria species exposed to multiple stresses. J. Basic Microbiol. 2009, 49, 593-597. [CrossRef] [PubMed]

43. Ramsey, M.M.; Freire, M.O.; Gabrilska, R.A.; Rumbaugh, K.P.; Lemon, K.P. Staphylococcus aureus Shifts toward Commensalism in Response to Corynebacterium Species. Front. Microbiol. 2016, 7, 1230. [CrossRef] [PubMed]

44. Jurek, I.; Góral, I.; Mierzyńska, Z.; Moniuszko-Szajwaj, B.; Wojciechowski, K. Effect of synthetic surfactants and soapwort (Saponaria officinalis L.) extract on skin-mimetic model lipid monolayers. Biochim. Biophys. Acta Biomembr. 2019, 1861, 556-564. [CrossRef] [PubMed]

45. Teng, Y.; Luo, Y.; Sun, M.; Liu, Z.; Li, Z.; Christie, P. Effect of bioaugmentation by Paracoccus sp. strain HPD-2 on the soil microbial community and removal of polycyclic aromatic hydrocarbons from an aged contaminated soil. Bioresour. Technol. 2010, 101, 3437-3443. [CrossRef] [PubMed]

46. Leow, Y.-H.; Maibach, H. Effect of occlusion on skin. J. Dermatol. Treat. 1997, 8, 139-142. [CrossRef]

47. GBD 2015 Disease and Injury Incidence and Prevalence Collaborators. Global, regional, and national incidence, prevalence, and years lived with disability for 310 diseases and injuries, 1990-2015: A systematic analysis for the Global Burden of Disease Study 2015. Lancet Lond. Engl. 2016, 388, 1545-1602. [CrossRef]

48. Bryld, L.E.; Agner, T.; Kyvik, K.O.; Brøndsted, L.; Hindsberger, C.; Menné, T. Hand eczema in twins: A questionnaire investigation. Br. J. Dermatol. 2000, 142, 298-305. [CrossRef]

49. Kampf, G.; Löffler, H. Prevention of irritant contact dermatitis among health care workers by using evidence-based hand hygiene practices: A review. Ind. Health 2007, 45, 645-652. [CrossRef]

50. Platts-Mills, T.A.E.; Erwin, E.; Heymann, P.; Woodfolk, J. Is the hygiene hypothesis still a viable explanation for the increased prevalence of asthma? Allergy 2005, 60, 25-31. [CrossRef] 